\title{
Textträger - Schriftträger: Ein Kurzportrait (statt Einleitung)
}

Der hier vorgelegte Band versammelt die Ergebnisse des Internationalen Kolloquiums „Textträger/Schriftträger“, das im Sommersemester 2010 am Altertumswissenschaftlichen Kolleg der Universität Heidelberg stattfand. Die Idee entstand in Heidelberg. Markus Hilgert und Diamantis Panagiotopoulos stellten das Thema „Text-Praxis“ zur Diskussion und luden im Rahmen des vierten Jahresvorhabens des Altertumswissenschaftlichen Kollegs den Kultursoziologen Andreas Reckwitz (damals Konstanz, jetzt Frankfurt/Oder) und die Historikerin Annette Kehnel (Mannheim) zur Zusammenarbeit ein.

Zur Debatte stand die Frage nach den Möglichkeiten von „Text-Handeln“, d. h. Handeln an, mit und infolge von Geschriebenem. Welcher Zusammenhang besteht zwischen Text und Handeln? Wie kann das reziproke Verhältnis, das Artefakte mit Sequenzen sprachlicher Zeichen und Akteure verbindet und sich im Handeln an, mit und infolge von Geschriebenem ausdrückt, beschrieben, konzeptualisiert und für die kulturwissenschaftliche Forschung fruchtbar gemacht werden? Dies war die vorgegebene Aufgabenstellung.

Ziel war es, die „praxisrelevante Effektivität des Geschriebenen“ in je spezifischen kulturellen Praktiken näher zu bestimmen und darauf aufbauend ein gemeinsames methodisches Instrumentarium und ein deskriptives Vokabular zu entwickeln, das im Sinne einer ,Text-Anthropologie‘ die bedeutungskonstitutiven ,Text-AkteurRelationen' darzustellen erlaubt (Hilgert 2010). Mit dem Brückenschlag zwischen historischer, empirischer und theoretischer Kulturwissenschaft sollte die Chance einer methodologischen und konzeptuellen Weiterentwicklung und damit die Realisierung jenes geisteswissenschaftlichen Forschungsverbundes vorangetrieben werden, der damals unter dem Arbeitstitel „Materialität und Präsenz des Geschriebenen in nontypographischen Gesellschaften“ an der Ruprecht-Karls-Universität Heidelberg aufgebaut und erfolgreich in den SFB 933 Materiale Textkulturen überführt wurde.

Andreas Reckwitz und Markus Hilgert forcierten die grundbegriffliche Klärung der Relation zwischen Praktiken, Diskursen und Artefakten mit dem Vorschlag, das Handeln an, mit und infolge von Geschriebenem als soziale Praktiken der Rezeption zu fassen (z. B. Aufschreiben, Lesen, Memorieren, Zitieren, Exzerpieren, Dramatisieren, Illustrieren, Kopieren, Kommentieren, Interpretieren, Verbergen, Re-Agieren). ,Texthandeln' wird so verallgemeinert zu schöpferischen Akten, in denen - je abhängig von konkreten sozialen und historischen Kontexten - mögliche ,Bedeutungen' des Geschriebenen konstituiert werden; schöpferische Akte die auf Wissensordnungen (Sinnmuster, Deutungsmuster, symbolische Codes) und subjektiven Sinnzuschreibungen basieren, die ihrerseits historisch und sozial-kulturell höchst variabel sind. 
Verbunden mit der Aufgabe der systematischen Rekonstruktion konkreter Rezeptionspraktiken wurde die Aussicht auf Grenzerweiterung. Die Aussicht auf Erweiterung der ,bedeutungsfixierten' Grenzen herkömmlich interpretativ-hermeneutischer Textanalyseverfahren.

Wir, Annette Kehnel und Diamantis Panagiotopoulos, die Herausgeber dieses Bandes, konkretisierten den Vorschlag in einem Gedankenexperiment zu den Möglichkeiten des „Handelns an, mit und infolge von Geschriebenem“ in Konzentration auf die schrifttragenden Artefakte in ihrer Qualität als Textträger bzw. Schriftträger. Dass Texte durch ihre Träger und Objekte durch ihre Beschriftung einen konkreten Sinn und symbolische Virulenz gewinnen, steht außer Zweifel. In der Phase ihrer Entstehung war die Schrift eng an Gebrauchsobjekte gekoppelt, indem sie als deren Etikett fungierte. Texte erfüllten ihre Funktion - ja ihren Sinn - nur in Verbindung mit den Gegenständen, die sie trugen. Später hat sich natürlich ein Großteil des Schrifttums als Kommunikationsmedium weitgehend von seinen Trägern verselbständigt - dies gilt insbesondere für die überwiegende Mehrheit der Texte, die wir als literarische Tradition einer Kultur erfassen können. Diese Texte wurden in der Regel auf neutralen Trägern weitertradiert oder gespeichert, die keine andere Funktion haben konnten, als einen Text aufzunehmen (Tontäfelchen, Pergament, Papyrus, Buch u. a.). Es war unvermeidlich, dass die wissenschaftliche (man könnte hier sagen die philologische) Auseinandersetzung mit Texten in verschiedenen Disziplinen auf das Geschriebene fokussierte und seinen Träger wegen seines neutralen Charakters, seiner eher unscheinbaren Materialität völlig ausgeblendet hat. Diese sehr einseitige Betrachtung von Texten wurde leider allzu häufig auch auf jene Texte angewendet, die auf Objekten mit einer eigenen, selbstständigen Gebrauchsfunktion angebracht waren (Architektur, Bildwerke, Grabsteine, Weihungen u. a.). Das internationale Kolloquium setzte sich daher zum Ziel, die Aufmerksamkeit auf das enge Verhältnis zwischen Text und Träger zu lenken. Aus verschiedenen Blickwinkeln sollte gezeigt werden, dass durch die Rückkopplung zwischen Texten und ihren Trägern, durch die Betonung ihrer Materialität und die Rekonstruktion ihres konkreten Wahrnehmungshorizonts in einem gegebenen historischen Kontext, ein wesentlicher Beitrag zum Verständnis sowohl vom Text als auch von dem ihn tragenden Artefakt geleistet werden kann. Bei diesem kontextimmanenten Ansatz geht es also um die Textur von Texten, ihre materielle Präsenz, die für ihr Verständnis genau so unverzichtbar ist wie ihr Inhalt.

Textträger tragen Text. Schriftträger tragen Schrift. Nimmt man dieses Bild wörtlich und lässt man der Vorstellung vom schrifttragenden Artefakt als handlungsfähigem Akteur freien Lauf, dann wird die Interaktion zwischen Geschriebenem und Beschriebenem zu einer treibenden Kraft im Spiel, dann öffnet sich der Vorhang einer Bühne, auf der historische Fallstudien vor den Kulissen dieses Gedankenexperimentes zur Aufführung gebracht werden können. Hier soll ,tragen' ganz konkret als eine Handlung in der primären Bedeutung des Wortes gefasst werden, d. h. als Aktivität, bei der etwas unter Einsatz physischer Kräfte gehalten, gestützt und/oder fortbewegt 
wird (nicht in verblasster Bedeutung von Verantwortung, Schuld oder Sorge tragen). ${ }^{1}$ Damit wird nicht allein die Materialität ins Bild geholt, sondern es wird auch das schrifttragende Artefakt zum Akteur: Als Text- und Schriftträger wird es handlungsfähig, muss die ihm anvertraute Last (Schrift, Text) tragen, halten, stützen, fortbewegen, wegschaffen, vielleicht auch loswerden, wird auf diese Weise zum Handlungsträger, fordert Aufmerksamkeit, evoziert Neugier, provoziert Aktionen und Reaktion etc., bringt - kurz gesagt - das hervor, was man materiale Textkulturen nennen könnte.

Wir fordern auf zum Spiel mit dem metaphorischen Überschuss im Bild des Schriftträgers. Dann nämlich kommt Bewegung ins Spiel. Rollentausch wird möglich und lenkt den Blick weg von der Fixierung auf die Vorstellung von Interaktion zwischen Schrift bzw. Text (passiv) und Rezipient (aktiv) im aktiv-passiv Modus hin zur Vorstellung eines Spiels zwischen gleichermaßen handlungs- und leidensfähigen Akteuren. Wenn schrifttragende Artefakte, personifiziert als Text- und Schriftträger, die Bühne betreten, wird die ihnen zugeschriebene Rolle als Handlungsträger anschaulich: Ihre Körper, ihr Aussehen, ihre Kostüme, ihre Bewegungen sind unmittelbar auf der Handlungsebene mit dem Spielgeschehen verknüpft. Im Bild gesprochen wird hier die Materialität der Beschreibstoffe - ihr Aussehen, die Beschaffenheit ihrer Oberfläche, ihr Gewicht, ihre Standorte, ihre Haltbarkeit etc. - in ihrer Bedeutung für die von ihnen hervorgebrachten Textpraktiken erkennbar.

Konkret mag dies an einem mittelalterlichen ,Stück vorgeführt werden, in dem ein Evangelienbuch als Textträger agiert. Das Buch, eine mittelalterliche Prachthandschrift, trägt in sich den Text der Evangelien. Sie wird von zwei Diakonen auf die Bühne getragen. Gespielt wird das Einsetzungszeremoniell eines mittelalterlichen Herrschers, konkret die Einsetzung des Bischofs von Rom als künftigem Papst, wie sie seit dem 6 . Jahrhundert n. Chr. praktiziert wurde. Während die anwesenden Bischöfe ihre Gebete sprechen, legen die Diakone das geöffnete Evangelienbuch - mit der Schrift nach unten - auf den Kopf bzw. auf den Nacken des Kandidaten (caput/cervix) in einem Akt, den die Einsetzungsliturgie als impositio evangeliorum bezeichnet. In diesem Akt der Auflegung wird die ,Schrift‘ zum Akteur, indem sie ihrerseits dem Kandidaten die Rolle des Schriftträgers aufzwingt. Sein Nacken muss sich strecken, Rücken und Knie müssen sich beugen, will er vermeiden, dass die ihm auferlegte Schrift zur Erde fällt. Wer die Position im Selbstversuch nachstellt, wird unmittelbar am eigenen Leib die Zumutung dieser vom Buch erzwungenen Haltung spüren. Der künftige Papst wird von der Materialität des ihm auferlegten Schriftträgers - vom Gewicht und von der Größe des prachtvoll eingebundenen Evangelienbuches - in einen Unterwerfungsgestus gezwungen. Dieser Zusammenhang kommt in außergewöhnlicher Anschaulichkeit in einer Darstellung der Einsetzung eines Papstes, vermutlich Papst Silvester II. im Sakramentar des Bischofs Warmund von Ivrea, entstanden um das Jahr 1000, zum Ausdruck. Das geöffnete Evangelienbuch,

1 DWDS 2014. 
zentral im oberen Drittel des Bildes, wird vom Diakon über den Kopf des Kandidaten gehalten, und es erscheint ein zweites Mal aufgelegt auf seinem Rücken (dem verlängerten Nacken), der sich unter der Last der Schrift nach vorne beugt. Hier wird anschaulich fassbar, wie die Materialität von Schrift und Text ihre eigenen Rezeptionspraktiken hervorzubringen in der Lage ist. Die dem Kandidaten auferlegte Rolle des Textträgers ist als spezifisch körpergebundene Rezeptionspraktik der Evangelien nicht unabhängig von deren Materialität vorstellbar. Es gab keinen Papst, der im Zuge seiner Einsetzung nicht von der „Schrift“ in die Knie gezwungen wurde.

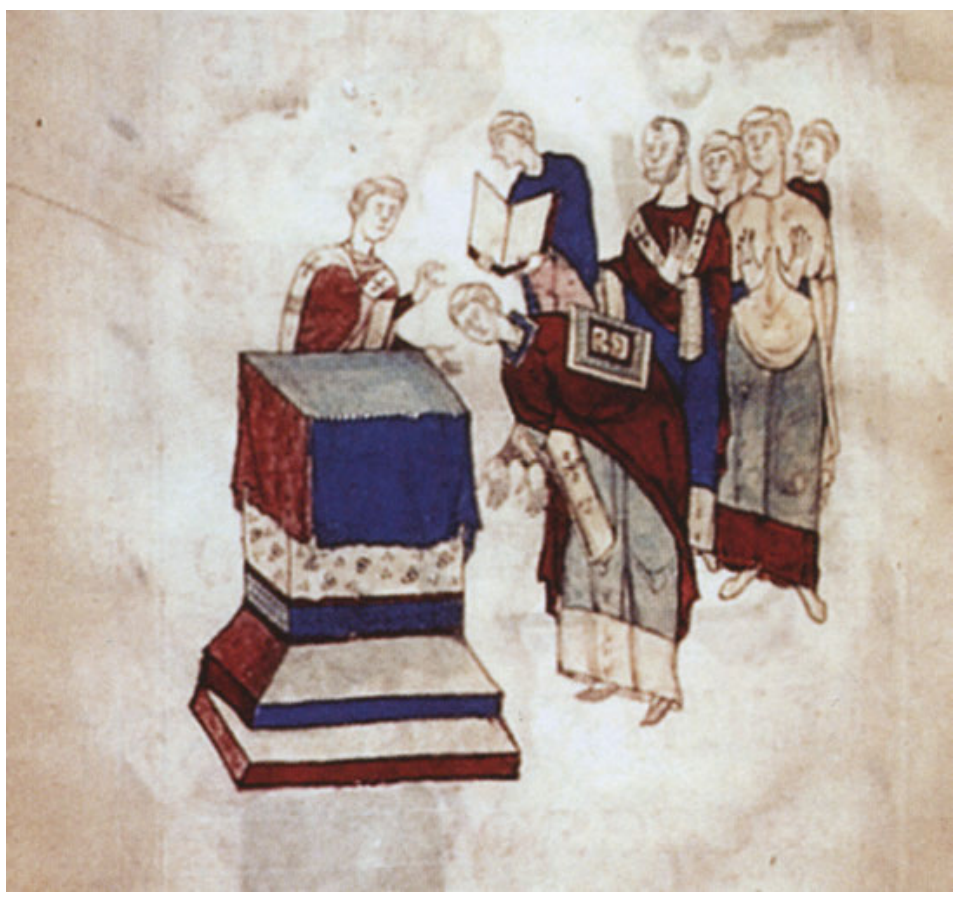

Abb. 1: Einsetzung (Ordination) des Papstes, vermutlich Papst Sylvester II. im Sakramentar des Warmundus von Ivrea, um 1000 (Detail). Ivrea, Biblioteca Capitolare, Ms. 31, LXXXVI, fol. 8r. (nach Mariaux 2002, Taf. 4).

Ein Zweites wird an diesem Beispiel sehr schön deutlich: die Fähigkeit der Schriftträger zur Verdoppelung von Wirklichkeit in der Verdoppelung ihrer Trägerfunktionen. So wie das Evangelienbuch seine Rolle als Schriftträger - vorübergehend im liturgisch gesicherten Rahmen - dem künftigen Papst aufzwingt, vermögen schrifttragende Artefakte in vielerlei Hinsicht ihre Trägerfunktion zu verdoppeln. Ihre Materialität birgt magische Kräfte. Sie können zaubern, machen ihre Umwelt zu Komplizen ihrer Textträgerschaft, machen Mitspieler zu Mitträgern, verwandeln andere in schrifttragende Artefakte und drängen wieder andere in die Rolle von Statisten. So 
jedenfalls scheint es, wenn man die Rolle der umstehenden Bischöfe im Sakramentar von Ivrea betrachtet.

Schrift- und Textträger verdoppeln die Wirklichkeit. Sie verfügen über transformative Kräfte. Sie üben Einfluss auf ihre Umwelt, sind Verwandlungskünstler, suchen sich Träger, wollen andere infizieren mit ihrer Trägerrolle. Sie funktionieren paradox. Agieren nicht nur, sondern gieren. Sie üben Einfluss nicht allein als Handelnde, sondern auch als Begehrende. Sie gieren nach Aufmerksamkeit, nach Berührung, nach Nähe, nach Distanz, erzwingen Handlungen, verunmöglichen dieselbe, provozieren und untersagen Bedürfnisse, fordern Bewegung oder verdammen zum Stillstand.

Textträger sind Machtträger. Sie üben Macht aus, indem sie Bedürfnisse erzeugen und die Aussicht auf Befriedigung unmittelbar mit ihrer Materialität verknüpfen: So lebt das Textamulett vom Schutzbedürfnis seines Trägers, das scheinbar leere Pergament einer Geheimschrift von der Neugier des Lesers, die unzugängliche Felsinschrift vom Verlangen des Verborgenen nach Unverfügbarkeit, die in den Grabstein gemeißelte Inschrift von der Sehnsucht der Vergänglichkeit nach Dauer etc.

Im oben erläuterten Gedankenexperiment hat sich nicht allein das schauspielerische Potential schrifttragender Artefakte gezeigt, das dann zum Tragen kommt, wenn sie in der Personifikation von Text- und Schriftträgern die Bühne betreten dürfen, sondern auch die Probleme der Fixierung auf all zu einsinnig vorgestellte Interaktionsmodelle. Phänomene der Schriftpräsenz lassen sich nur sehr begrenzt als Interaktionsform zwischen - tendenziell eher passiv vorgestellter - Schrift und aktiven Rezipienten fassen. Schrifttragende Artefakte sind nicht auf Rezeptionspraktiken angewiesen, ganz im Gegenteil: sie bringen diese selbst hervor. Scherben, Rotuli oder Grabsteine in der Rolle der Schriftträger, die personifizierte Vorstellung aktiver, handlungs- und leidensfähiger Teilhaber an kulturellen Praktiken, schafft Distanz zu den überkommenen bedeutungsfixierten Interpretationsgewohnheiten im Umgang mit Schrift und Text. So wird Raum geschaffen für neue Versuchsanordnungen, die Möglichkeit zum „Umparken im Kopf“. Anders gesagt: Die metaphorischen Überschüsse der Schriftträger-Bildlichkeit setzten Kreativität frei, sie erlauben ein Nachdenken über Artefakte - über Haut, Papier, Gewebe, Grenzsteine, Fluchtafeln, Amulette, Vasen, Felswände, Rinden etc. - nicht als Speicher von in Schrift gefasstem ,Sinn“, von kulturellem Wissen, dessen Bedeutung von anderen (Rezipienten) abgerufen werden muss oder kann, sondern als handelnde Subjekte, deren Handlungsfähigkeit mit einer körperlichen ,Macht‘ verknüpft ist, die Wirklichkeiten hervorzubringen, zu transformieren und auszulöschen vermag. Eben dieser transformativen Macht spüren die im vorliegenden Band versammelten Beiträge nach, indem sie in je spezifischen Einzelstudien zu höchst unterschiedlichen schrifttragenden Artefakten - von der Tonscherbe bis zur Sammelhandschrift, vom Buchamulett bis zur Hundeleine - nach der Vielfalt kultureller Deutungsangebote fragen, nach Gebrauch und Umgang der Schriftträger mit ihren Körpern. Diese Einzelstudien versuchen die neuen Ansätze 
zur Materialität und Präsenz der Schrift aus verschiedenen Perspektiven, in verschiedenen historischen Kontexten und mit verschiedenen Methoden für ein neues Verständnis von Texten und text- bzw. schrifttragenden Artefakten fruchtbar zu machen. Sie werden hier in einer thematischen Reihenfolge vorgelegt, an deren Anfang zwei für das Forschungskonzept des SFB 933 grundlegende Texte (Lieb und Ott, Feraudi) stehen. Daran schließen sich Beiträge an, die nach den unterschiedlichen Formen von Materialität bzw. Praxis geordnet sind und sich mit ,getragener' Schrift (Kopp, Berlejung, Skemer), Schrift auf Pergament (Kössinger, Müller), Schrift auf Fels bzw. Ton (Fitzenreiter, Gerleigner) und schließlich Schrift auf Haut (Richter, Kehnel) auseinandersetzen.

Ludger Lieb und Michael Ott (beide Heidelberg) behandeln erzählte oder fiktionale Schriftträger, die in mittelalterlichen Texten vorkommen. Anhand von konkreten Fallbeispielen wird hier versucht, das komplexe Verhältnis von Text, Artefakt, Produzent und Rezipient zu ergründen. Diese Beispiele umfassen: 1) die Erzählung von Progne und Philomela in Albrechts von Halberstadt Übertragung der Metamorphosen Ovids, bei der die Schrift auf einem weißen Tuch (Ovid) bzw. einem weißen Gürtel (Albrecht von Halberstadt) als Substitut der mündlichen Rede fungiert, 2) die Tafel des Gregorius (aus Hartmanns von Aue Gregorius), eine Wachstafel, die engstens mit der Biographie des Protagonisten dieser Geschichte verwoben ist, 3) das schrifttragende Hundehalsband (,Brackenseil') im Titurel Wolframs von Eschenbach, das einen Hund mit seiner Leine zum Schriftträger macht und schließlich 4) die Passage aus der Vita Heinrich Seuses, in der der Protagonist den Namen Jesu in die Haut über seinem Herzen ritzt. In all diesen Fällen geht es um mobile und potentiell mobilisierbare schrifttragende Artefakte, die vielfältige und spannungsbeladene Kommunikationskanäle ermöglichen und etablieren. Bei ihrer Behandlung übernehmen die beiden Autoren die von Konrad Ehlich eingeführten Begriffe ,lokomobil' und ,lokostatisch'. Die ,lokomobilen' Artefakte, die im Mittelpunkt dieses Beitrags stehen, sind schrifttragende Artefakte, die Schrift von einem Ort zu einem anderen bringen. Durch den Transport eines Textes in neue Handlungskontexte werden sowohl seine Bedeutung als auch insgesamt die Grenzen der schriftlichen Kommunikation wesentlich erweitert. Diese transportablen und portablen Artefakte werden schließlich einem Fall von immobilen Schriftträgern gegenübergestellt: die Bauminschriften fiktionaler Schäfer in Martin Opitz' Schäfferey von der Nimfen Hercinie, die als Einritzungen in lebendigen Oberflächen eine gewisse Lebendigkeit innehaben. In all diesen mittelalterlichen Texten wird die enge Verklammerung zwischen Artefakt, Schrift und Mobilität, die vielfältige Kommunikationsmodi eröffnet, explizit.

Francisca Feraudi-Gruénais (Heidelberg) fragt nach der Rolle des Textträgers in der Epigraphik und stellt rezeptionspraktische Text-Akteur-Relationen an verschiedenen Beispielen vor. An den Anfang ihrer Überlegungen stellt sie - in gewollter Provokation - einen Käse, dessen Rinde eine Aufschrift trägt. Was ist wichtiger, der 
(Schrift-) Gebrauch auf der Rinde oder der Inhalt? Damit ist die „Gretchenfrage“ an den Doktor Faust der materialen Textkulturen formuliert: „Nun sag, wie hast du's mit dem Inhalt, mit dem Sinn, mit der Bedeutung?“ Ist die Bedeutung des Textträgers wichtiger als der Inhalt des Textes? Wo bleibt der Sinn? Antworten auf diese Frage sucht der Beitrag in zahlreichen Fallstudien, deren anschaulichste wohl die antiken Wurfgeschosse aus Blei (glandes plumbeae) sein dürften, beschriftet mit Flüchen oder bösen Wünschen, wie etwa der, die Kugel möge den Hintern des Feindes treffen. Ohne den mobilen Textträger wäre eine solche Inschrift zwar nicht ungefährlich, aber deutlich weniger wirksam. Der Text verdankt seine Wirksamkeit der Materialität seines Trägers. In ihrem Plädoyer für das Kerngeschäft der Epigraphik, nämlich die Re-Kontextualisierung von Inschriften unter Einbeziehung von Einzelerkenntnissen zu greifbaren Objekten, Spuren von Raumordnungen und Reflexen dynamischer Phänomene (z. B. Rituale), wird deutlich, dass epigraphische Forschung über Fragen zu Text-Textträger-Relationen hinaus gehen, und diese zwar wichtig, aber nur ein Aspekt unter vielen anderen sein kann.

Amina Kropp (Mannheim) stellt die Rolle von Bleitäfelchen als Textträger im kulturellen Gebrauch sogenannter defixio-Rituale zur Diskussion. Über ein Jahrtausend vom 6. vorchristlichen bis ins 5. nachchristliche Jahrhundert ist diese magische Praktik durch mehr als 500 erhaltene - überwiegend bleierne - Fluchtäfelchen belegt. Kropp umschreibt das Ritual als eine Zauberhandlung zur Lösung „drängender persönlicher Angelegenheiten“ mit der eine andere Person beeinflusst, bezwungen, erobert oder bestraft werden konnte. Zu diesem Zweck wurden dünne Metallplättchen mit Verwünschungen beschriftet (Schadens- und Zwangszaubern), durchbohrt oder auf andere Weise manipuliert und dann an Orte mit unheilvoller Macht, wie etwa Gräber oder unbenutzte Brunnen getragen und abgelegt. Die Metalltäfelchen fungieren zunächst als Stellvertreter für die Zielperson des Zaubers sodann als Träger der Verwünschung zur rituellen Manipulation, sie werden anstelle des Opfers mit dem Fluch belegt, durchbohrt, malträtiert. Über den Textträger werden die gewünschten Folgen für das Opfer gleichsam analog materialisiert, wie z. B. in folgendem Fluch: „Wie dieses Blei nicht auftaucht und untergeht, so soll untergehen seine Jugend, seine Gliedmaßen, sein Leben [...]“. Kropp weist auf die Leistung dieser schriftvermittelten Kommunikation hin, die in der Materialität der Fluchtafel die raumzeitliche Trennung von Sender und Empfänger zu überbrücken weiß. Vom Wissen um die zentrale Bedeutung der Materialität des Beschreibstoffes für die Wirksamkeit des Zaubers zeugt folgende Gebrauchsanleitung im sogenannten ,Homerischen Dreizeiler‘: „Für eine Offenbarung: schreib auf ein Lorbeerblatt [...]. Um Rennwagen zu stürzen, räuchere [...] Knoblauch [...]. Für Bannungen schreib auf eine Meermuschel [...]. Um Gunst zu erwerben [...]: schreib auf ein Goldtäfelchen [...]. Bei herbeizwingenden Liebeszaubereien: räuchere Rose und Sumach [...]“ (vgl. Kropp, Anm. 24). In Konzentration auf das Wechselspiel zwischen Text und Textträger diskutiert der Beitrag Fragen der materialgebundenen Wirksamkeit der Bleitäfelchen und ihre Funktion als Katalysatoren für die 
Performativität des Rituals und kommt mit Isabel Zollna zu folgendem Schluss: „Die Wahrnehmung von Schrift auf ihrem materiellen Träger als bloßes Medium ist eine Reduktion, die sich in einer bestimmten Gesellschaftsform entwickelt hat.“

Angelika Berlejung (Leipzig) fragt nach dem Gebrauch und der Wirkung von Textamuletten im Alten Orient (Mesopotamien, Syrien, Ägypten und der Levante). Im Gegensatz zum antiken Fluchtäfelchen wird im Amulett stets die Gegenwart einer Gottheit repräsentiert, die den Träger schützt und trägt. Amulette waren mobile, materiale und performative Zeichen der Gottgegenwart, durch die der Träger göttliche Gegenwart in sein Alltagsleben holte und dämonische Kräfte aus demselben bannte. Berlejung prägt den Begriff der portativen Gottespräsenz, die durch die Alltagstauglichkeit der bequem tragbaren Amulette ermöglicht wird. Im Bezug auf spezifische Besonderheiten von Textamuletten lassen sich rollsiegel- oder tonbullenartige Perlen oder Gemmen, die man auffädeln konnte, Röllchen aus dünnem Metallblech oder Papyrus, die in Kapseln mit Aufhängevorrichtung aufbewahrt wurden und kleine Schreibtäfelchen aus Ton, Stein oder Metall mit Aufhängevorrichtung unterscheiden. Die Texte sind zum einen Beschwörungen, die den Amulettträger vor Unglück, Krankheiten, bösen Träumen bzw. vor den verursachenden Dämonen schützen sollten. Zum anderen finden sich apotropäische Gebete, $d$. h. Texte, die die Götter ermuntern und dazu auffordern, dem Amulettträger beizustehen und sich zu seinen Gunsten einzusetzen. Besondere Aufmerksamkeit widmet der Beitrag der praktischen Frage, wie und wo am Körper die Textamulette getragen wurden, nämlich auf der Brust in unmittelbarer Nähe des Herzens. Hier kommt dem alttestamentlichen Auftrag an die Israeliten, sich die Worte des Mose auf Herz, Hand und zwischen die Augen (= auf die Oberstirn) zu binden besondere Bedeutung zu. Berlejung vermutet, dass es sich hier um konkrete Objekte mit Textaufschrift handelt, die auf (!) dem Herzen zu tragen waren. Nach Moses Worten sollte das Gesetz jedem Menschen auf den Körper gebunden sein. Auf diese Weise ließ sich eben auch theologisch-ethische Programmatik in den Alltag des einzelnen Menschen einbringen. Die Zitation biblischer Verse in hebräisch-aramäisch-samaritanischen Textamuletten oder Zauberschalen sind aus späteren Zeiten durchaus bekannt. Da die Textamulette dauernd am Körper getragen wurden, waren sie zwar körperfixierte, aber mobile und alltagsfähige Dauerbegleiter des Menschen, zeitlebens mit ihrem Träger in einem unmittelbaren, persönlichen, körperlichen Verhältnis verbunden bis in sein Grab.

Don C. Skemer (Princeton), setzt die Geschichte des Gebrauchs von Textamuletten ins westeuropäische Mittelalter fort. Obgleich vergleichsweise wenige Funde aus dem Mittelalter erhalten sind, vor allem weil sie meist am Körper getragen und nicht in Schränke oder Kisten verwahrt wurden, blieben die wenigen erhaltenen Stücke weitgehend unerforscht. Theologen und Rechtshistoriker sahen in ihnen Zeugnisse primitiven mittelalterlichen Aberglaubens, obwohl, und das wird in Skemers Studie sehr deutlich, die Gläubigen und auch der Klerus regelmäßig legitimen Gebrauch von 
Textamuletten machten. Im 12. Jahrhundert zum Beispiel trug einer der Baumeister von Norham Castle stets ein seidenes Säckchen mit einem Stück Pergament bei sich, auf dem die Namen Christi und der Prolog aus dem Johannesevangelium geschrieben waren. Ein Mönch aus Durham bot ihm eine kleine Reliquie aus dem Heiligtum von St. Cuthberth zur Ergänzung an. Der Text eines weiteren, sehr interessanten Amuletts aus dem 13. Jahrhundert, heute in der Canterbury Cathedral Library, MS Additional 23, erinnert den Träger acht mal an die Vorteile davon, mächtige Worte und Schriften bei sich (super se) zu tragen. Ein Textamulett für den familiären Gebrauch versichert seinem Träger, dass er vor Ertrinken, Tod durch Feuer und vorschnellem Tod verschont bliebe, dagegen mit erfolgreichen Niederkünften gesegnet werde. Daneben gibt es auch spezielle Geburtsamulette zum Gebrauch für Schwangere kurz vor und während der Niederkunft. Sie wurden an Gürteln getragen und auch über den Bauch der Gebärenden gelegt. Dass die Dominikaner in San Jacopo di Ripoli in Florenz einen florierenden Handel mit Textamuletten betrieben, bestätigt ebenfalls die Vereinbarkeit mittelalterlicher Frömmigkeit und Textamulettgebrauch. Skemer kommt zu dem Schluss, dass Textamulette in der longue durée zu den beständigsten Begleitern der Menschheit zählen dürften. Er identifiziert sie als festen Bestandteil der materiellen Kultur des homo portans seit der Erfindung der Schrift durch Antike und Mittelalter bis heute. Die Produktion und der Gebrauch von Textamuletten wurde über tausende von Jahren und trotz Veränderungen in ihren Inhalten, Methoden der Produktion und der Beschreibmaterialien kontinuierlich gepflegt. Sie blieben vergleichsweise immun gegen sich ändernde geopolitische Realitäten, florierten in religiösen Synkretismen und überlebten die europäische Aufklärung und das Zeitalter der modernen Wissenschaften bis in den Cyberspace des 21. Jahrhundert, wo Internet-Anbieter in Second Life Textamulette als Protektionszauber an Mitspieler in Not verkaufen.

Norbert Kössinger (Wien) untersucht die funktionalen Potentiale ,gerollter Schrift“ am Beispiel mittelhochdeutscher Texte auf Rotuli. Schrift auf aneinander genähten Pergament- oder Papierstreifen, die anders als Schrift auf den Seiten eines Kodex nicht durch Aufschlagen und Blättern, sondern durch Aufrollen lesbar wird. Anhand ausgewählter Beispiele aus seiner Sammlung von mehr als 35 Rotuli mit deutschsprachigen Texten aus verschiedensten Bereichen (von medizinischen Texten über Verwaltungsschriftum und liturgischen Texten bis zu Dichtung und Chronistik) zeigt Kössinger zunächst die produktionstechnische Flexibilität als maßgeblichen Vorteil der Schriftrolle gegenüber dem Buch. Der sogenannte Rotulus von Mülinen, eine fast sechs Meter lange und 13 Zentimeter breite Schriftrolle, besteht aus insgesamt 15 Streifen unterschiedlichster Länge, die von 5 Zentimeter bis zu fast einem Meter reichen und die aneinander genäht oder geklebt wurden. Es scheint, dass diese Rolle kontinuierlich verlängert wurde und zwar in einem Arbeitsprozess, der mehr als 50 Jahre umfasste. Das kontinuierliche Sammeln der enthaltenen Gebrauchstexte (460 lateinische Rezepte, Beschwörungen und Segensformeln, botanisches Wissen und ein Pflanzenglossar) wird wesentlich von der Schriftrolle als Textträger gefördert. Serielle 
Anschlüsse lassen sich hier leichter herstellen als beim Codex, wenn er einmal mit zwei Buchdeckeln versehen ist. Am Beispiel des Osterspiels von Muri kann Kössinger die Vorteile der Schriftrolle im Aufführungsgebrauch schön veranschaulichen: Die Rolle in der Hand des Souffleurs bei der Probe und Aufführung des Spiels. Die Schriftrolle erlaubt „visuelle Kopräsenz“ von Text, die im engen Korsett von Versound Recto-Seiten eines Codex nicht möglich ist. Sie erlaubt ,Scrollen' statt ,Blättern' was in konkreten Aufführungssituationen, wie dem des geistlichen Spiels, praktische Vorzüge bietet. Drittens ist gerollte Schrift beweglicher und mobiler als codexgebundene Schrift. Viertens wurden Rollen für genau determinierte Kontexte produziert, nämlich Gebrauchssituationen und Sprechsituationen, die in der Nähe von mündlicher Kommunikation und Formen von Textvortrag stehen.

Stephan Müller (Wien) erklärt am Beispiel des ,Trierer Teufelsspruches` warum mittelalterliche Geheimschriften keine Geheimschriften sind. Nicht Verschleierung der Lesbarkeit eines Textes - sei es zum Zwecke der Geheimhaltung, aus Spielerei oder als Ausdruck von Gelehrtenstolz - sei Sinn und Zweck der Geheimschrift, sondern vielmehr geht es darum, einem Text eine besondere Form zu geben, die von der erwarteten Form abweicht. Das kann ganz unspektakulär der Fall sein, wenn man Text und Paratext trennt; das ist brisanter, wenn man damit eine Art spezieller Verfügungsgewalt über einen Text zur Schau stellt und seine eigene Schrifterfindung feiert. Schließlich gipfelt es in Formen, Texten zusätzliche Bedeutungsdimensionen zuzuschreiben, die ihnen die Niederschrift in Klarschrift nicht mitgeben konnte. Geheimschrift wird somit $\mathrm{zu}$ einer Form der Textträgerschaft, die die Rezeption des Geschriebenen maßgeblich bestimmt: Das Lesen wird verlangsamt, es ist nicht möglich einen Text flüchtig, en passant wahrzunehmen. Oder, positiv formuliert, der Leser wird in den Text hineingezogen, der Text erschließt sich ihm in anderer Form als der der gewohnten Lektüre. Müller geht noch weiter mit der Vermutung, dass die durch die Geheimschrift erzwungene Verlangsamung in der Entzifferung einen Textes eine Form der ,offenbaren“ oder meditativen Aneignung fördere. Die Geheimschrift lenkt die Aufmerksamkeit auf den Text, sie bedingt eine konzentrierte und vermeidet eine flüchtige Form der Lektüre und bindet den Rezipienten so stärker an den Text, als das eine Aufzeichnung in Klarschrift täte. Und, um es auf einen Punkt zu bringen: Die Form der Schrift stattet den Spruch mit einer zusätzlichen Bedeutungsdimension aus und schreibt ihm eine besondere Geltung zu, die mit einer besonderen Form der Rezeptionspraxis korrespondiert.

Martin Fitzenreiter (Münster) nähert sich dem Phänomen der scheinbaren Verbergung von bedeutungscodierenden Medien im pharaonischen Ägypten. Seine Forschung konzentriert sich auf Schriften, die an intentionell unzugänglichen Orten - wie dekorierte Innenseiten von Särgen, Fugeninschriften, Totentexte in der Sargkammer oder schwer zugängliche Felswände - angebracht wurden. Diese ,unzugänglichen' Schriften wurden nicht grundlos produziert, sondern bewusst unzu- 
gänglich gehalten, sowohl was den Anbringungs- und Aufbewahrungsort, aber auch was die kodierte Botschaft selbst angeht. Statt nach dem Inhalt dieser verborgenen Texte fragt Fitzenreiter nach den Trägermedien, die die entsprechenden Aufschriften und Bilder konserviert haben und zugleich deren Eigenschaft der Unzugänglichkeit bestimmen. Zum anderen interessieren ihn die Prozesse von Produktion, Wahrnehmung und möglicher Rezeption, die sich um diese Trägermedien modellieren. Er geht vom ,Primat der Artefakte‘ gegenüber ihren möglichen Inhalten aus und davon, dass die Kehrseite des Bemühens um die Verborgenheit der Schriften die Präsenthaltung des Wissens um ihre Existenz war. Die Materialität des Geschriebenen garantiert das Wissen um die Schrift ,im Verborgenen'. Dabei reicht das Wissen, dass dort im Verborgenen Objekte existieren, die entsprechende Texte und Bilder tragen. Das Wissen um die Existenz sinntragender Objekte setzt die Kenntnis wesentlicher Sinnzusammenhänge voraus, die mit dem Objekt des Text- und Bildträgers einerseits, dem Inhalt der Botschaft andererseits verbunden sind.

Im Akt der Anbringung werden die Potenzen der eingesetzten Trägermedien aktiviert (der Berg, die Schrift, der Name, das Gold etc.) und die konkrete Aussage des Textes konstituiert sich im Moment der Herstellung, wenn die Auftraggeber und Produzenten sozusagen selbst zu Rezipienten der von ihnen erstellten Botschaft werden. „Denn so merkwürdig es klingen mag: nicht für die Ewigkeit und nicht für irgendwelche irrationalen Rezipienten wird (praktisch gesehen) diese Inschrift angebracht, sondern für den Moment ihrer Anbringung und ihre Rezeption durch jene, die sie anbringen (lassen).“

Georg Simon Gerleigner (Kiel) stellt griechische Vasen als Text-Bild-Träger zur Diskussion. Sein Beitrag zur Schriftbildlichkeit, anschaulich gemacht im Bild der Schrift als Ejakulat auf einer attischen Strickhenkelamphora, geht einher mit einer grundlegenden Kritik des Logozentrismus im Umgang mit Schrift und Text. Denn der Logozentrismus, so Gerleigner, reduziert den Rezipienten auf die Rolle des ,Lesers' und sorgt dafür, dass die großartige Pointe der Textbildinschrift ignoriert wird, die nämlich, dass die Platzierung und der graphische Verlauf der in einem Bogen zu Boden schießenden Buchstaben suggerieren, dass sie ejakuliert werden bzw. Ejakulat darstellen! Die Markierungen mit dem Schlicker können nicht nur als Buchstaben gelesen, sondern auch und gleichzeitig als Körperflüssigkeit gesehen werden: „Zweifach sehen die, die Schriftzeichen gelernt haben“. Einerseits erzielte dieser raffinierte visuelle Kniff einen weiteren witzigen Effekt, während er andererseits die Aufmerksamkeit auf den Textinhalt der Inschrift zog und die ,Signatur' dadurch mit dem offensichtlichen Esprit (oder der Schöpfungskraft) ihres Urhebers verband, was wiederum die werbende Wirkung seiner (stolzen) Aussage - „Smikros hat’s gemalt!“ - verstärkte. Der Vasenmaler zeigt damit auch, dass er mit dem Medium Schrift, gerade in seiner speziellen Kombination mit dem Medium Bild, umzugehen versteht. 
Die abschließenden Beiträge zu diesem Band beschäftigen sich mit der menschlichen Haut als Textträger. Susan Richter (Heidelberg) verfolgt spätaufklärerische Diskurstraditionen zu Schrift auf Haut, zur Interpretation der Kulturtechnik des Tätowierens, wie sie etwa in James Cooks Reiseberichten über die Südseeinsulaner nach Europa gelangten. Der Untersuchungszeitraum fällt in die Zeit um 1800, in der - in Anlehnung an Hans Ulrich Gumbrecht - neue anthropologisch-philosophischästhetischen Beobachtungsverfahren emergieren. In diesem Zeitraum, in dem für Gumbrecht die „epistemologische Moderne“ mit noch üblichen Reflexionsmustern ihren Anfang nahm, wurden innerhalb früher anthropologischer Wissenschaftskonzepte der Mensch als Natur- oder Kulturwesen diskutiert. Dabei rückten Körper (und Seele) als Wissensorgane in das Blickfeld von wissenschaftlicher und selbstreflexiver Betrachtung. Auch Interaktionen zwischen Körper und Seele standen zur Debatte. In dem Zusammenhang wurden Sprache und Schrift im Verhältnis zum Körper - etwa im Sinne von Einschreibeverfahren - diskutiert sowie Ausdrucksmöglichkeiten bzw. die Zeichenhaftigkeit von Körpersprache als Ausdruck des lesbaren Körpers untersucht. Unmittelbar anschaulich wird diese in Goethes Reflexionen über die Analogie zwischen Namen und Haut:

... denn der Eigenname eines Menschen ist nicht etwa wie ein Mantel, der bloß um ihn her hängt und an dem man allenfalls noch zupfen und zerren kann, sondern ein vollkommen passendes Kleid, ja wie die Haut selbst ihm über und über angewachsen, an der man nicht schaben und schinden darf, ohne ihn selbst zu verletzen.

Richters Studie verfolgt insbesondere zeitgenössische Diskussionen über die rechtshistorischen Implikationen der Kulturtechnik des Tätowierens, die in der Verwendung des Tattoos als Rechtsdokument sichtbar wird. Sie erlauben es, den indigenen, mit Schrift tätowierten Körper als Ausdruck seiner Rechtsfähigkeit, seiner rechtlichen Abhängigkeiten, Verpflichtungen oder Verbindlichkeiten sowie seiner Rechte und Pflichten in der Gemeinschaft zu interpretieren. Die Körper der gesamten lebenden Gemeinschaft bildeten somit einerseits eine Abbildung geltenden Rechts bzw. geltender Gesetze sowie die Verankerung des Einzelnen darin - etwa durch die Dokumentation seiner Vergehen oder seines Einklangs mit dem Gesetz. Die Haut avancierte zum Wissensspeicher, zum Archiv der Identität seines Trägers. Jedoch zu einem im Gegensatz zu den verschlossenen europäischen Archiven, wo das Archiv selbst Dokument und dieses sofort les- und erfassbar für alle Mitglieder der Gemeinschaft blieb - Dokument das mit dem Tod seines Trägers erlischt. Haut fungierte somit als begrenztes Gedächtnis von Ordnungen mit befristetem Erinnerungswert.

Annette Kehnel (Mannheim) fragt nach Vorstellungen vom Menschen als ,Textträger' im kollektiven Metaphernhaushalt abendländischer Traditionen. Der mit Noten beschriftete und gemarterte Mensch im Garten der Lüste des Hieronymus Bosch, der mit der Heilsgeschichte beschriebene gemarterte Köper des Gekreuzigten Christus und 
schließlich der mit dem Evangelienbuch belastete Körper des Papstes bieten reichlich Anschauungsmaterial für Vorstellungen und kulturelle Praktiken des mit Schrift belasteten Körpers. Der Beitrag erkundet den metaphorischen Mehrwert im Bild des Menschen als Textträger und die Implikationen für ein Verständnis des Lebens als Text (Schrift, Buch) der sich in die Körper seiner Träger kontinuierlich einschreibt.

\section{Literaturverzeichnis}

DWDS (2014): Digitales Wörterbuch der deutschen Sprache (Berlin-Brandenburgische Akademie der Wissenschaften) http://www.dwds.de, Stand: 29.4.2014

Hilgert (2010): Markus Hilgert, ,,Text-Anthropologie‘: Die Erforschung von Materialität und Präsenz des Geschriebenen als hermeneutische Strategie“, Mitteilungen der Deutschen Orientgesellschaft 142, 87-126.

Mariaux (2002): Pierre Alain Mariaux, Warmond d'lvrée et ses images. Politique et création iconographic autour de l'an mil, Bern. 
